\title{
Gene Therapy: Applications, Insights and Prospects
}

\author{
Tayebeh Sanchooli ${ }^{1,{ }^{*}}$ and Mahtab Teimouri ${ }^{2, * *}$ \\ ${ }^{1}$ Department of Anatomical Sciences, School of Medicine, Zahedan University of Medical Sciences, Zahedan, Iran \\ ${ }^{2}$ Department of Anatomical Sciences, School of Medicine, Shahid Beheshti University of Medical Sciences, Tehran, Iran \\ "Corresponding author: Department of Anatomical Sciences, School of Medicine, Zahedan University of Medical Sciences, Zahedan, Iran. Email: tasanchooli@gmail.com \\ ${ }^{* *}$ Corresponding author: Department of Anatomical Sciences, School of Medicine, Shahid Beheshti University of Medical Sciences, Tehran, Iran. Email: \\ mahtab.teymuri@yahoo.com
}

Received 2021 September 26; Revised 2021 October 16; Accepted 2021 October 25.

Keywords: Somatic Gene Therapy, Gene Transfer, Gene Therapy

\section{Dear editor,}

Gene therapy is known as a technique of delivering the genetic material to the patient's body to correct the altered genes or site-specific modifications that have therapeutic effects (1). Gene therapy is divided into two categories of somatic gene therapy (SGT) and germline gene therapy (GGT) (2).

SGT is a condition in which the therapeutic genes are transferred into somatic cells. Any modification cannot pass to the next generation, and the offspring of the patient can develop the disease (1). Two techniques are used in SGT, ex vivo and in vivo. In the first type, cells are cultured outside the body, exposed to the viral vector for genetic modification, and then, transferred to the patient's body. Another type is based on the direct administration of the vector to the cells of the body (3) (Figure 1).

GGT focuses on germ cells (oocytes and sperms) or the preimplantation embryo. In this method, the germ cells are modified by the entrance of functional genes into their genome. This causes permanent changes that are inherited by all next generations (4).

One of the main aspects of gene therapy is the optimization of delivery vehicles (vectors) that are mostly plasmids, nanostructured, or viruses. The viruses are the best candidate for gene delivery due to their excellence in invading cells and inserting their genetic material (1).

Gene therapy is considered a promising approach in the treatment of various diseases, such as autoimmune diseases, diabetes, cancers, and heart diseases that cannot be treated using conventional methods (5).

The US Food and Drug Administration (FDA) approved the first gene therapy approach, which was conducted in 1989. In this study, immunotherapy was performed for patients with advanced melanoma (6).
In 2017, the US FDA approved Voretingene voretigene (Luxturna, Spark Therapeutics Inc) for the treatment of RPE65-associated leber congenital amaurosis (LCA) (7).

Type 1 diabetes mellitus (T1DM) is an autoimmune disease characterized by destroying insulin-secreting islet $\beta$ cells mediated by $\mathrm{T}$ cells. Manipulating of responsible genes of this disease by gene therapy could help manage the disease and even cure T1DM(5). Rao et al. demonstrated that the transfer of the hepatocyte growth factor (HGF) genes using adenovirus into rodents' pancreatic islets increased the expression of these genes. Overexpression of HGF induced the $\beta$ cells proliferation and enhanced their survival and functions (8).

Gene therapy seems to be an opportunity to improve the treatment of inherited monogenic retinal disorders. In most clinical studies, recombinant adeno-associated virus (AAV) or lentivirus vectors have been used for gene transfer to the retinal cells. RPE65 is one of the genes, which their mutations cause LCA. A lack of functional RPE65 leads to the inability of the rod photoreceptor cells to respond to light. Several trials have reported that subretinal injection of a recombinant AAV2/2 vector containing the RPE65 cDNA can enhance retinal function and vision in humans with RPE65$\operatorname{LCA}(7)$.

Cancer treatment using gene therapy has been increased in recent decades. Various gene therapy approaches were employed for cancer management, including cancer- and/or tumor-specific promoters, including cancer suppressor genes, anti-angiogenic gene therapy, suicide gene therapy, immunotherapy, etc. (9).

Carcinoembryonic antigen (CEA) belongs to a cellsurface glycoproteins family and is considered as a tumor marker in the clinical diagnosis of several cancers, such as colorectal cancer (CRC). It is overexpressed in more than 


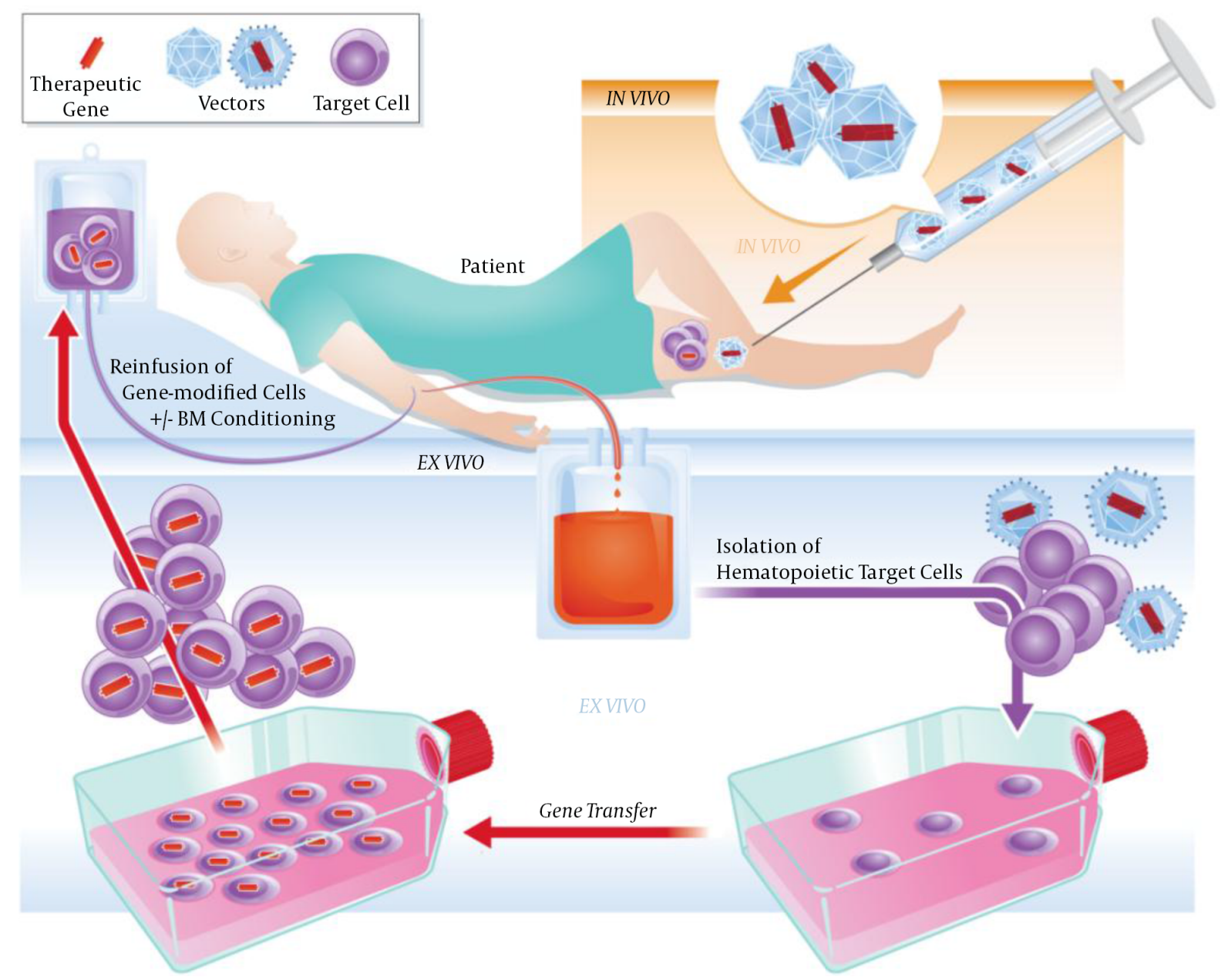

Figure 1. In vivo and ex vivo gene therapy concepts (2). In the in vivo technique, the therapeutic gene is introduced directly into the body, while in the ex vivo, the patient's cells are extracted from the body, genetically modified outside the body, and then transferred into the patient.

90\% of CRC cells. Its promoter has been used in directing suicide $E$ gene (pCEA-E) against colon cancer in order to high suppression of cell growth in CRC cells compared to normal colon cells (10).

Neurodegenerative disorders are another candidate for gene therapy. Several studies have demonstrated that the transfer of adeno-associated viruses- glial-derived neurotrophic factor (AAV-GDNF) in the putamen is safe and effective for treating Parkinson's disease in murine models (11). Also, a clinical trial using bilateral AAV2- aromatic L-amino acid decarboxylase (AADC) injection in the putamen of severely affected patients with Parkinson's disease reported similar results (12).

In gene therapy, a normal gene is transferred to the genome to replace an abnormal gene that caused a specific disease. Various challenges are involved in this process, in- cluding lack of adequate information about the genes involved in a certain disease, especially complex diseases, in which both genetic and environmental factors play a role. Also, a potential immune response to AAV vectors is controversial.

Despite these limitations, further progression in the safe and effective vector design, identifying the specific cells of the body that need treatment, and a better understanding of the pathomechanisms of diseases and their genetic issues will be an open window in genome editing as a new treatment approach for untreatable diseases.

In conclusion, gene therapy can be employed to complement conventional therapies resulting in more effective treatments. 


\section{Footnotes}

Authors' Contribution: T.S contributed to the study concept, drafting of the manuscript, and revision of the manuscript for important intellectual content. M.T contributed to the drafting of the manuscript.

Conflict of Interests: The authors declared no conflicts of interests.

Funding/Support: This study was supported by the Zahedan University of Medical Sciences.

\section{References}

1. Goncalves GAR, Paiva RMA. Gene therapy: advances, challenges and perspectives. Einstein (Sao Paulo). 2017;15(3):369-75. doi: 10.1590/S1679-45082017RB4024. [PubMed: 29091160]. [PubMed Central: PMC5823056].

2. Kaufmann KB, Buning H, Galy A, Schambach A, Grez M. Gene therapy on the move. EMBO Mol Med. 2013;5(11):1642-61. doi: 10.1002/emmm.201202287. [PubMed: 24106209]. [PubMed Central: PMC3840483].

3. Fraldi A, Serafini M, Sorrentino NC, Gentner B, Aiuti A, Bernardo ME. Gene therapy for mucopolysaccharidoses: in vivo and ex vivo approaches. Ital J Pediatr. 2018;44(Suppl 2):145-54. doi: 10.1186/s13052018-0565-y. [PubMed: 30442177]. [PubMed Central: PMC6238250].

4. Wolf DP, Mitalipov PA, Mitalipov SM. Principles of and strategies for germline gene therapy. Nat Med.2019;25(6):890-7. doi:10.1038/s41591019-0473-8. [PubMed: 31160821].
5. Chellappan DK, Sivam NS, Teoh KX, Leong WP, Fui TZ, Chooi K, et al. Gene therapy and type 1 diabetes mellitus. Biomed Pharmacother. 2018;108:1188-200. doi: 10.1016/j.biopha.2018.09.138. [PubMed: 30372820].

6. Rosenberg SA, Aebersold P, Cornetta K, Kasid A, Morgan RA, Moen R, et al. Gene transfer into humans-immunotherapy of patients with advanced melanoma, using tumor-infiltrating lymphocytes modified by retroviral gene transduction. N Engl J Med. 1990;323(9):570-8. doi: 10.1056/NEJM199008303230904. [PubMed: 2381442].

7. Kumaran N, Michaelides M, Smith AJ, Ali RR, Bainbridge JWB. Retinal gene therapy. Br Med Bull. 2018;126(1):13-25. doi: 10.1093/bmb/ldy005. [PubMed: 29506236].

8. Rao P, Cozar-Castellano I, Roccisana J, Vasavada RC, Garcia-Ocana A. Hepatocyte growth factor gene therapy for islet transplantation. Expert Opin Biol Ther. 2004;4(4):507-18. doi: 10.1517/14712598.4.4.507. [PubMed:15102600].

9. Belete TM. The Current Status of Gene Therapy for the Treatment of Cancer. Biologics. 2021;15:67-77. doi: 10.2147/BTT.S302095. [PubMed: 33776419]. [PubMed Central: PMC7987258].

10. Montano-Samaniego M, Bravo-Estupinan DM, Mendez-Guerrero O, Alarcon-Hernandez E, Ibanez-Hernandez M. Strategies for Targeting Gene Therapy in Cancer Cells With Tumor-Specific Promoters. Front Oncol. 2020;10:2671. doi: 10.3389/fonc.2020.605380. [PubMed: 33381459]. [PubMed Central: PMC7768042].

11. Axelsen TM, Woldbye DPD. Gene Therapy for Parkinson's Disease, An Update. J Parkinsons Dis. 2018;8(2):195-215. doi: 10.3233/JPD-181331. [PubMed: 29710735]. [PubMed Central: PMC6027861].

12. Eberling JL, Jagust WJ, Christine CW, Starr P, Larson P, Bankiewicz $\mathrm{KS}$, et al. Results from a phase I safety trial of hAADC gene therapy for Parkinson disease. Neurology. 2008;70(21):1980-3. doi: 10.1212/01.wnl.0000312381.29287.ff. [PubMed: 18401019]. 